\section{Case Reports in Ophthalmology}

Case Rep Ophthalmol 2020;11:582-587

\title{
A Case Report of Diffuse Lamellar Keratitis after FemtoSMILE Refractive Surgery
}

\author{
Saeed S. Alqahtani \\ Department of Surgery, Faculty of Medicine, Najran University, Najran, Kingdom of Saudi \\ Arabia
}

\section{Keywords}

Diffuse lamellar keratitis · Small-incision lenticule extraction · SMILE operation

\begin{abstract}
Diffuse lamellar keratitis (DLK) is not an uncommon complication of the small-incision lenticule extraction (SMILE) operation. Instrumental causes, such as contamination, are usually the underlying etiological route. Herein, we present an atypical case of DLK with numerous foci of cellular infiltrates on day 1 . No evidence of any diffuse inflammatory response was reported. On the other hand, the multifocal response began to improve starting on the third day, after the administration of systemic steroids, with pain and discomfort significantly disappearing on the second day. Antibiotics were given as well, in the case of secondary infections. After 1 week of treatment and close monitoring, significant improvement was reported, and the slit-lamp examination was unremarkable.

(C) 2020 The Author(s)

Published by S. Karger AG, Basel
\end{abstract}

\section{Introduction}

In the fall of 1997 in the American Academy of Ophthalmology meeting in Chicago, an informal report was made by Maloney and Smith, describing a "self-limited" noninfectious inflammatory phenomenon, occurring at the interface of the multiple layers of collagen fibrils 


\section{Case Reports in Ophthalmology}

(i.e., lamellae) of the human corneal stroma, following the LASIK procedure within a short period $[1,2]$.

Diffuse lamellar keratitis (DLK) was first known as the "shifting sands phenomenon" or "sands of Sahara syndrome," owing to its whitish, coarse appearance comprising currents of inflammatory cells infiltrating the cornea [2]. Wide variations exist between the epidemiological reports concerned with this condition. The incidence of DLK in patients undergoing LASIK surgery, shortly after the procedure, has been found to range from nearly 0.1 to $20 \%$. Studies investigating this complication rate in patients with epithelial defects have found that $90 \%$ or more were affected in a nonoutbreak setting. Conversely, in outbreaks, a more generalized etiology would seem reasonable for the escalation of these case clusters. For instance, contamination of steam sterilizers with endotoxins as well as marking surgical pens and gloves were all found to be highly influential in the spread of outbreaks in epidemic areas [3-7].

As first described by Linebarger et al. [8], the staging system of DLK was found to be helpful, not only in determining the prognosis of the condition but also in choosing the correct treatment regimen for each stage. Based on the severity and location of the inflammatory reaction, DLK can be categorized into 4 stages: stage 1, in which the whitish granular pattern can be seen extending in the lamellar periphery, sparing the visual axis and requiring thorough inspection for detection on day 1 postoperatively; stage 2, where the granular pattern is seen shifted to the central part of the corneal lamella, reaching the visual axis and giving the occasionally observed shifting-sand appearance, which can be detected on the second and third postoperative days; stage 3 , which is the stage of intensification of the granular reaction in the lamellar center, causing a marked reduction in visual acuity, and stage 4 that refines the end result of severe, uncontrolled DLK, where stromal scarring massively impairs vision [8].

On the other hand, small-incision lenticule extraction (SMILE) operations can yield complications like DLK [9]. Interestingly, unlike the LASIK surgery, DLK caused by SMILE operations might have some distinguishing features, such as the absence of the diffuse cellular infiltrate, with only widely scattered focal lesions as the main criterion of this unusual presentation [10]. In this report, we present the case of a 29-year-old patient with an atypical presentation of DLK following SMILE operation.

\section{Case Report}

A 29-year-old male patient was seen in October 2017 at our institution for corneal assessment before laser refractive surgery. The patient's manifest refractions of the right and left eyes were -3.5 sphere and -3.25 sphere, respectively, with a 20/20 corrected distance visual acuity (CDVA) in both. The central corneal thickness of the right eye was $525 \mu \mathrm{m}$, and it was $522 \mu \mathrm{m}$ in the left eye.

The SMILE procedure was done using the Visumax femtosecond laser (Carl Zeiss Meditec AG). The cap diameter was $7.2 \mathrm{~mm}$, with an intended thickness of $130 \mu \mathrm{m}$, and the optical zone in both eyes was $6.3 \mathrm{~mm}$. No intraoperative complications were reported. Plastic shields were prescribed during sleep for the first 3 nights postoperatively. Moxifloxacin drops (Vigamox) were used for the first 10 days and prednisolone acetate (Pred Forte) for the first 7 days, 4 times a day, then tapering the dose during the last 3 weeks.

On the first day, mild discomfort in both eyes, more lateralized to the left eye, was reported. The uncorrected distance visual acuity (UDVA) was 20/40 in both eyes. A slit-lamp examination of the left cornea showed multiple white foci within the corneal lamellar interface, as shown in Figure 1. Although infection was a possible explanation for this observation, 
the probability of a noninfectious inflammatory reaction outbalanced that of an infection, owing to the multiplicity of the lesions and their early onset. Accordingly, moxifloxacin (Vigamox) drops were given in a thrice-daily dose and continued throughout the study to avoid the catastrophic consequences of a potential microbial infection. Furthermore, topical prednisolone acetate 1.0\% (Pred Forte) was given every hour. Further reassessment was done on the next day.

On the second day, the patient reported the pain of discomfort to have "disappeared," with slight blurring, more in the left eye. The UDVA in both eyes was 20/40. Slit-lamp examination showed similar, yet slightly milder, findings to those observed on the first day. Due to the persistent inflammatory reaction, steroidal preparations were a reasonable choice of treatment at that time; hence, the patient was started on an oral steroid at $80 \mathrm{mg}$. The patient was asked for a revisit the next day for further examination.

On the third postoperative day, the patient reported feeling further improvement with a reduction in the "amount of blur on the left side." In the right eye, the UDVA was 20/20, while it was 20/22 in the left eye. Slit-lamp examination of the right eye showed much less-dense infiltrates than those on the first 2 days. Meanwhile, in the left eye, mild restoration of the normal appearance was observed, as shown in Figure 2. Another visit was planned on the next day for more follow-up.

On the fourth day, with a UDVA of 20/20 in the right eye and 20/22 in the left, the slitlamp examination showed the same findings as on day 3 . As a result, we decided to decrease the dose of both Pred Forte and the oral steroid to 1 drop every $2 \mathrm{~h}$. The patient was asked to return after 2 days.

On the sixth day, when observed with the slit lamp, both eyes started to show marked clearance of the infiltrates. In the right eye, the UDVA was $20 / 20$, while it was $20 / 20^{-2}$ in the left eye. By that time, the next step was to reduce Pred Forte to a 4-times-per-day dosage and tap the oral steroid over the next 3 days by $20 \mathrm{mg}$ a day.

One week later (day 13), the patient mentioned feeling great with sharp vision in both eyes. The UDVA was 20/20 in both eyes with normal intraocular pressure, which was $16 \mathrm{~mm}$ $\mathrm{Hg}$ in both globes. Clear corneas were seen under the slit lamp. The patient was instructed to taper the dose of Pred Forte down to thrice daily during the following week and for each of the next 3 weeks.

After 5 weeks of observation and in-concordance management (day 34), the infiltrates had successfully resolved in both eyes (Fig. 3). UDVA was 20/20, and the intraocular pressure was $18 \mathrm{~mm} \mathrm{Hg}$ in both eyes. Slit-lamp examination was completely normal. Lubricant drops were prescribed, and follow-up in 4-6 months was advised.

A routine follow-up was performed after 6 months, without any further reported complaints or complications. At UDVA of 20/20 in both eyes, the autorefraction was Plano in the right eye +0.25 and +0.50 in left. The latest slit-lamp examination showed a clear cornea (Fig. 4). Normal corneal topography was found in both eye globes.

\section{Discussion}

As regards the inflammatory cellular infiltrates, this is considered a rare case of DLK, in which a pattern of widely dispersed or disseminated reactionary infiltrates is lacking, giving a characteristic multifocal appearance unusual for typical DLK cases. To our knowledge, only 1 previous case report of noninfectious multifocal inflammatory lamellar keratitis has been published [10]. One challenging aspect of this atypical presentation of DLK is the increased 


\section{Case Reports in Ophthalmology}

probability of microbial infections, whose clinical picture and slit-lamp examination overlap with those of the current case. In other words, in this type of DLK cases, infections must always be included in the differential diagnosis, and the patient must undergo treatment accordingly. Consequently, microscopic and cultural samples must be retrieved from patients with such presentations to exclude any evidence of the presence of microorganisms and avoid the severe complications that could result. In another context, the standard treatment protocol for DLK is hourly topical steroidal preparations under strict monitoring. This is done by irrigating the corneal lamellar interface after lifting the corneal cap. Moreover, oral steroids are a plausible treatment option and can be used as prophylaxis [11-14]. However, steroid-induced glaucoma can result due to excessive use. Other complications of DLK include corneal scarring and loss of best-corrected visual acuity [14, 15].

In conclusion, DLK is usually caused by contaminated instruments and occurs in the form of outbreaks. Such etiological factors must be identified and resolved instantly to avoid the emergence of an epidemic. Some cases of DLK may present with an atypical picture that lacks the dense, diffuse component of a typical DLK presentation. It is necessary to include infections in the differential diagnosis of these cases. Consequently, antibiotic eye drops are an obligatory treatment. DLK cases usually resolve through intensive administration of corticosteroids, which should be given under close observation to avoid induced glaucoma.

\section{Statement of Ethics}

The author of this work declares that this experiment complied with the guidelines for human studies and was in accordance with the World Medical Association Declaration of Helsinki. Written informed consent was obtained from the patient for publication of this case report and any accompanying images. Any sort of information that might reveal the patient's own identity has been completely avoided.

\section{Conflict of Interest Statement}

The author has no conflicts of interest to declare.

\section{Funding Sources}

The author did not receive any form of research grant, financial support, or funding for this study.

\section{Author Contributions}

The author confirms sole responsibility for the following: study conception and design, data collection, analysis and interpretation of results, and manuscript preparation. 


\section{Case Reports in Ophthalmology}

\section{References}

1 Smith RJ, Maloney RK. Diffuse lamellar keratitis. A new syndrome in lamellar refractive surgery. Ophthalmology. 1998 Sep;105(9):1721-6.

2 Linebarger EJ, Hardten DR, Lindstrom RL. Diffuse lamellar keratitis: identification and management. Int Ophthalmol Clin. 2000;40(3):77-86.

3 Gritz DC. LASIK interface keratitis: epidemiology, diagnosis and care. Curr Opin Ophthalmol. 2011 Jul;22(4):251-5.

4 Holland SP. Update in cornea and external disease: solving the mystery of "sands of the Sahara" syndrome (diffuse lamellar keratitis). Can J Ophthalmol. 1999 Jun;34(4):193-4.

5 Villarrubia A, Palacín E, Gómez del Río M, Martínez P. Description, etiology, and prevention of an outbreak of diffuse lamellar keratitis after LASIK. J Refract Surg. 2007 May;23(5):482-6.

6 Hoffman RS, Fine IH, Packer M, Reynolds TP, Bebber CV. Surgical glove-associated diffuse lamellar keratitis. Cornea. 2005 Aug;24(6):699-704.

7 Lazaro C, Perea J, Arias A. Surgical-glove-related diffuse lamellar keratitis after laser in situ keratomileusis: long-term outcomes. J Cataract Refract Surg. 2006 Oct;32(10):1702-9.

8 Linebarger EJ, Hardten DR, Lindstrom RL. Diffuse lamellar keratitis: diagnosis and management. J Cataract Refract Surg. 2000 Jul;26(7):1072-7.

9 Zhao J, He L, Yao P, Shen Y, Zhou Z, Miao H, et al. Diffuse lamellar keratitis after small-incision lenticule extraction. J Cataract Refract Surg. 2015 Feb;41(2):400-7.

10 Stuart A, Reinstein DZ, Vida RS, Archer TJ, Carp G. Atypical presentation of diffuse lamellar keratitis after small-incision lenticule extraction: sterile multifocal inflammatory keratitis. J Cataract Refract Surg. 2018 Jun;44(6):774-9.

11 Cosar CB, Sener AB, Sen N, Coskunseven E. The efficacy of hourly prophylactic steroids in diffuse lamellar keratitis epidemic. Ophthalmologica. 2004 Sep-Oct;218(5):318-22.

12 Peters NT, Lingua RW, Kim CH. Topical intrastromal steroid during laser in situ keratomileusis to retard interface keratitis. J Cataract Refract Surg. 1999 Nov;25(11):1437-40.

13 MacRae SM, Rich LF, Macaluso DC. Treatment of interface keratitis with oral corticosteroids. J Cataract Refract Surg. 2002 Mar;28(3):454-61.

14 Shah MN, Misra M, Wihelmus KR, Koch DD. Diffuse lamellar keratitis associated with epithelial defects after laser in situ keratomileusis. J Cataract Refract Surg. 2000 Sep;26(9):1312-8.

15 Lyle WA, Jin GJ. Interface fluid associated with diffuse lamellar keratitis and epithelial ingrowth after laser in situ keratomileusis. J Cataract Refract Surg. 1999 Jul;25(7):1009-12.
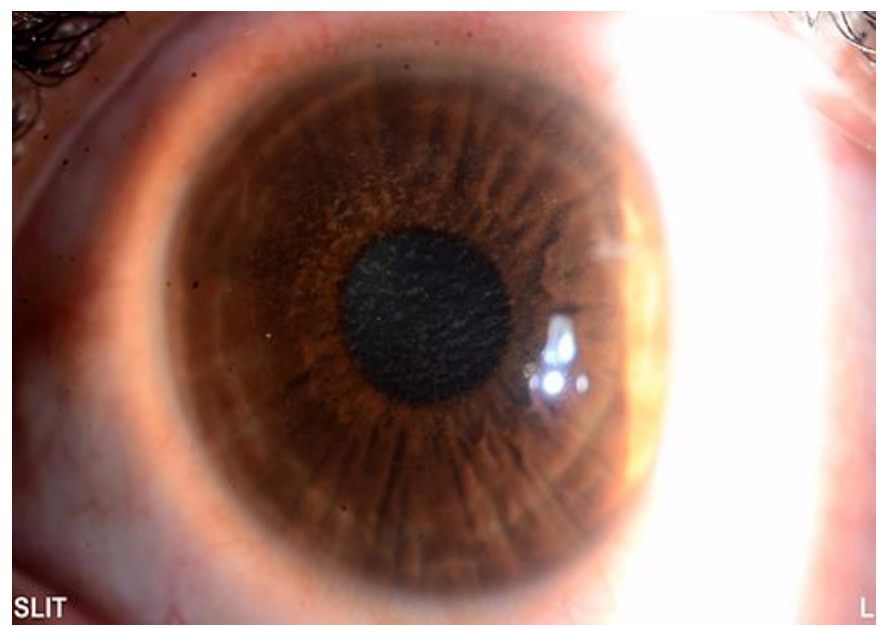

Fig. 1. Multiple scattered focal white infiltrates. 
Case Reports in Ophthalmology

Case Rep Ophthalmol 2020;11:582-587

(c) 2020 The Author(s). Published by S. Karger AG, Basel DOI: $10.1159 / 000509851$ www.karger.com/cop

Alqahtani: A Case Report of Diffuse Lamellar Keratitis after FemtoSMILE Refractive Surgery

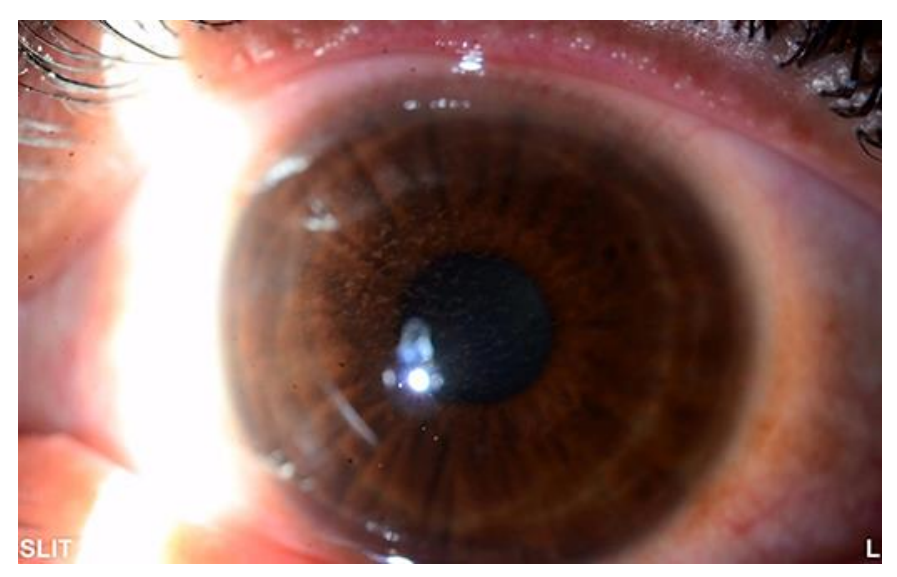

Fig. 2. Less-dense infiltrates.

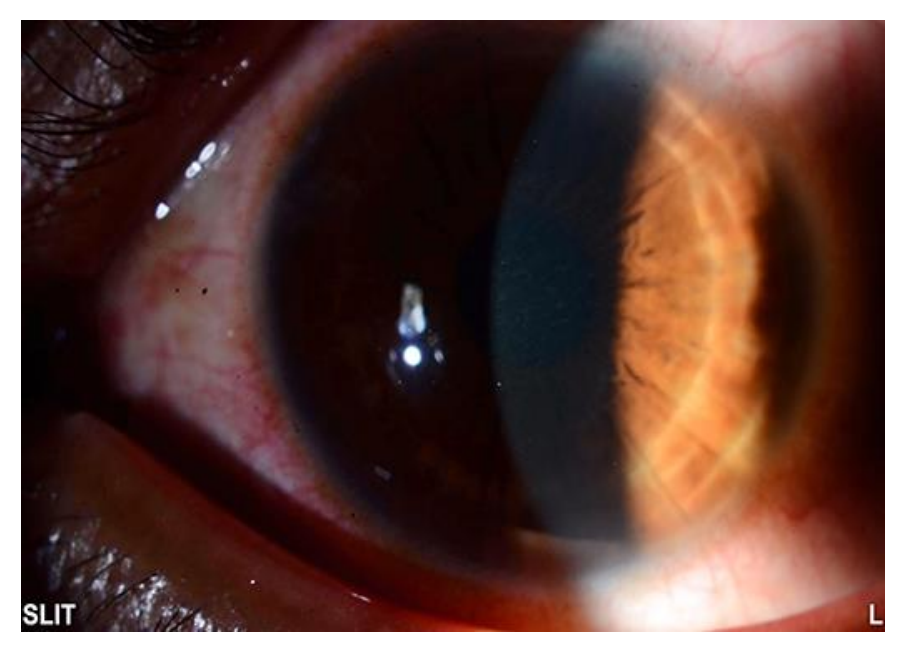

Fig. 3. Infiltrates have entirely resolved.

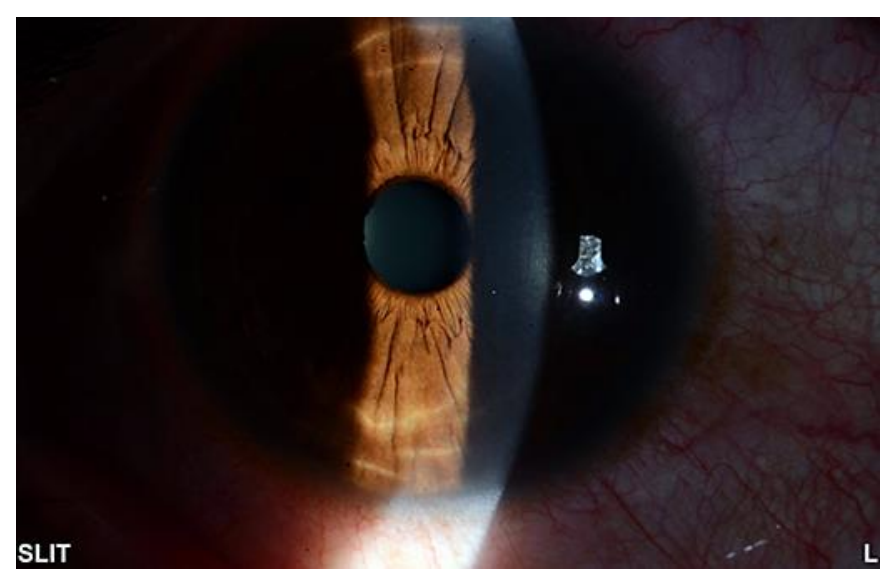

Fig. 4. Clear cornea. 